\title{
Precise optical characterization of SNAP structures with a reference fiber
}

\author{
ARtemiy V. DMitriev ${ }^{1, *}$ AND M. Sumetsky ${ }^{1}$ \\ ${ }^{1}$ Aston Institute of Photonic Technologies, Aston University, Birmingham B4 7ET, UK \\ *Corresponding author: a.dmitriev@aston.ac.uk
}

Compiled December 8, 2016

\begin{abstract}
A method of precise characterization of Surface Nanoscale Axial Photonics (SNAP) structures with a reference fiber is proposed, analyzed and demonstrated experimentally. The method is based on simultaneous coupling of a microfiber to a SNAP structure under test and to a reference optical fiber. Significant reduction of measurement errors associated with the environmental temperature variations and technical noise of the spectrum analyzer is demonstrated. The achieved measurement precision of the effective radius variation of the SNAP structure is $\mathbf{0 . 2} \AA$. (๑) 2016 Optical Society of America
\end{abstract}

OCIS codes: (060.2340) Fiber optics components; (140.3945) Microcavities; (230.3990) Micro-optical devices.

http://dx.doi.org/10.1364/ao.XX.XXXXXX

Surface Nanoscale Axial Photonics (SNAP) is a microphotonic platform which allows one to create photonic structures at the surface of an optical fiber with unprecedented sub-angstrom precision [1-5]. The propagation of whispering gallery modes (WGMs) in the axial direction of SNAP structures is fully controlled by small $\left(\sim 10^{-5}\right)$ variation of fiber radius and refractive index along the SNAP structure, which can be described by a single parameter, the effective radius variation (ERV). This nanoscale variation can be introduced by local partial annealing of the fiber with focused $\mathrm{CO}_{2}$ laser radiation [1, 4], femtosecond laser inscription [2], local heating [3] or piezoelectrically induced mechanical stress [6]. The measurement of ERV is performed using a biconical taper with a microfiber waist (a microfiber, for brevity), which is translated along the SNAP structure and connected to the optical spectrum analyzer (Fig. 1(a)) [7, 8]. The ultrahigh fabrication precision, ultrahigh measurement precision, and ultralow loss of silica fibers, which are combined in SNAP technology, potentially enable the creation of new generation of miniature slow light photonic devices (e.g., delay lines, optical buffers and processors [5,9]) not possible previously [10, 11].

Ultrahigh fabrication precision of SNAP structures requires the development of a robust and accurate method for their characterization. In particular, the acquired optical spectra should not be affected by the temperature variation in the process of measurement (which may take hours) and the apparatus noise. The current characterization method illustrated in Fig. 1(a) does not satisfy these criteria. For example, for a silica fiber with radius of $\sim 50 \mu \mathrm{m}$, the variation of temperature $\sim 0.10 \mathrm{~K}$ causes the ERV of $\sim 0.5 \AA$, which exceeds the currently achieved fabrication precision of $0.2 \AA$ [4].

Several methods can be implemented to take into account the thermal drift in the process of characterization of microresonators. For example, resonant frequencies of different optical modes depend on the temperature of the microresonator in different ways. Therefore, it is possible to determine the temperature drift from the change of the resonant frequencies of two optical modes with different mode indices. Consequently, the effect of the temperature variation can be excluded by introducing the temperature-dependent corrections into the experimentally measured spectra $[12,13]$. Alternatively, coupling microresonators to atomic systems allows one to use the atomic transitions as temperature-independent frequency reference sources [14, 15].

However, these methods cannot be directly applied to the characterization of SNAP devices, in which the effective radius varies along the fiber. The precision of the characterization of SNAP devices is also affected by the random technical drift of the reference frequency source of the measuring device (spectrum analyzer). Therefore it is desirable to have a method that allows one to eliminate the effect of technical and thermal drift during the measurements of the spectra of SNAP structures.

In this letter a method of spectral characterization of SNAP structures using an additional reference fiber is proposed and realized experimentally. This allows one to exclude the influence of the temperature drift as well as of the technical frequency noise of the spectrum analyzer. The letter is organized as follows: (i) the general principles of the proposed referencing method are explained; (ii) the experimental setup is described, (iii) the practical aspects and limitations of the realization of the proposed method are detailed and (iv) the results of the characterization of a SNAP bottle resonator are presented and discussed.

The proposed characterization method, which is illustrated in Fig. 1(b), involves simultaneous characterization of the SNAP fiber under test and a very smooth and accurately characterized reference fiber. The fibers are positioned parallel to each other at a small distance to minimize the temperature difference between them. This distinguishes the proposed approach from that suggested in [16], where the reference microresonator was stationary. Light is coupled to both fibers simultaneously with a normally aligned microfiber, which is connected to an optical spectrum analyzer. The microfiber is translated along the fibers 


\section{(a)}

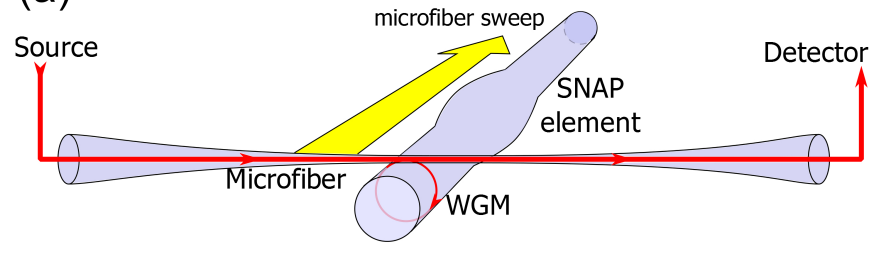

(b)

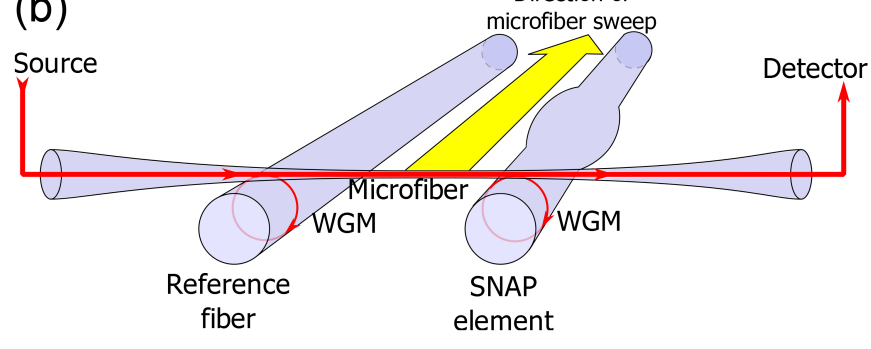

(c)

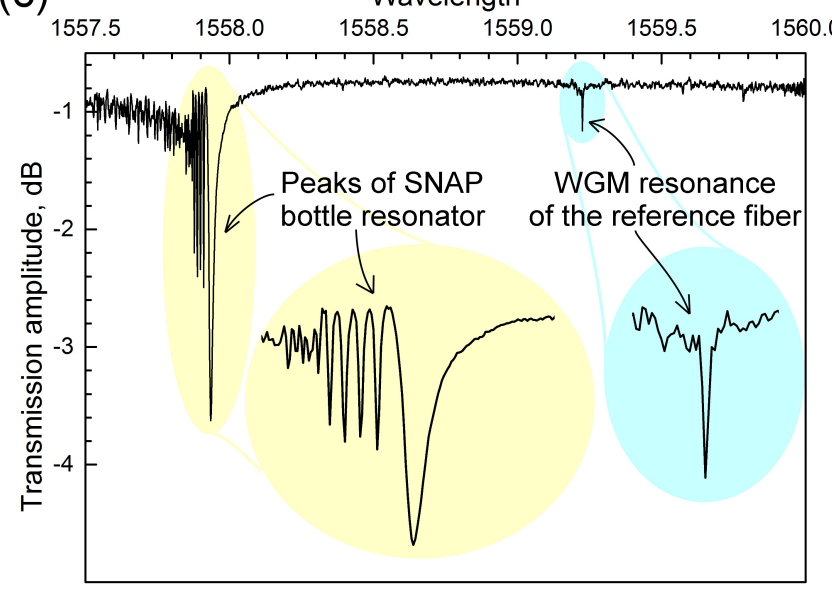

Fig. 1. Measurements of the ERV of a SNAP structure: (a) standard schematic $[7,8]$ and (b) the proposed method with a reference fiber. Characterization is performed by sweeping tapered microfiber along the SNAP structure. (c) Typical measured transmission spectrum of the microfiber, which includes both the WGM resonances of the SNAP structure under test and the reference resonant peak corresponding to a WGM of the reference fiber.

and touches them at contact points, where the transmission spectrum is measured. In logarithmic scale, this spectrum is the superposition of spectra of each of these fibers. For this reason, the resonant peaks corresponding to the WGMs of the both fibers are present in the measured spectra simultaneously (Fig. 1(c)). This allows for reducing the effect of the ambient temperature drift and the apparatus noise by referencing the spectrum of the structure under test to the spectrum of a uniform fiber. The measured spectra are processed as follows. For each spectrum, the reference peak (shown, e.g., in a blue circle in Fig. 1(c)) and its central wavelength are identified. The frequencies in each spectrum part that describes the SNAP structure are then shifted by the value of the determined change in reference resonant wavelength.

The SNAP structure under test is fabricated of a $38 \mu \mathrm{m}$ diameter silica fiber. The reference fiber is a standard $125 \mu \mathrm{m}$ diameter single-mode optical fiber, which has a smaller ERV per

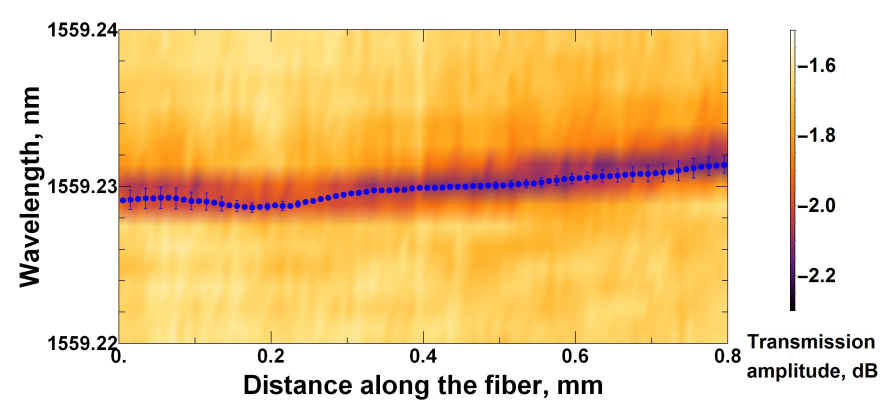

Fig. 2. Calibration of the reference. The averaged dependence of the reference peak (black dots with error bars) on the distance along the fiber over 3 different measurements is plotted over one of the measured surface plots of the transmission amplitude (color saturation) through the microfiber.

unit length and a higher Q-factor than those from which the SNAP structure is made. The ends of the fiber under test and the reference fiber are fixed on a rigid supporting structure with epoxy. The distance between the reference fiber and the fiber under test is equal to $1.5 \mathrm{~mm}$. The microfiber with the waist of $1.6 \mu \mathrm{m}$ in diameter is placed normal to the fibers and connected to an optical spectrum analyzer (LUNA OVA 5000). The position of the microfiber relative to the fibers is controlled by 3-axis remotely controlled translation stages. During the characterization process the microfiber is translated along the fiber under test and the reference fiber at relatively large distance $(>0.1 \mathrm{~mm})$. This translation is periodically interrupted and the microfiber is brought into direct contact with the fibers to measure the transmission spectrum. The direction of the microfiber can be slightly adjusted so that the microfiber touches either the SNAP fiber under test or the reference fiber. This is used in preliminary measurements to distinguish the resonances of the SNAP fiber and those of the reference fiber. The wavelength range of the measured spectra is set to $2.55 \mathrm{~nm}$. The resolution of the spectrum analyzer is $1.6 \mathrm{pm}$. The time of a single spectrum measurement is about $0.5 \mathrm{~s}$; the period between consecutive measurements is $10 \mathrm{~s}$. Thus the overall speed of the characterization process is $1 \mathrm{~mm}$ per $5000 \mathrm{~s}$ for the spatial resolution of $2 \mu \mathrm{m}$. At each measurement, the obtained spectrum is calibrated with respect to the value of transmission amplitude in an off-resonance spectral region. This reduces the influence of the random contamination of the microfiber on the measured spectra.

In the first order of perturbation theory the shift $\Delta \lambda_{r}$ of the resonant wavelength $\lambda_{r}$ with temperature change $\Delta T$ can be expressed as

$$
\frac{\Delta \lambda_{r}}{\lambda_{r}}=\frac{\Delta n}{n}+\frac{\Delta r}{r}=\left[\frac{1}{n}\left(\frac{\partial n}{\partial T}\right)+\alpha\right] \Delta T .
$$

Here $n$ is the refractive index, $\partial n / \partial T$ and $\alpha$ are the thermooptic coefficient and the coefficient of thermal expansion of the fiber's material, respectively $\left(\partial n / \partial T \approx 1.3 \times 10^{-5} \mathrm{~K}^{-1}\right.$ and $\alpha \approx 5.5 \times 10^{-7} \mathrm{~K}^{-1}$ for fused silica at room temperature). From Eq. (1) it follows that the shift of the resonant wavelength is independent on the fiber's radius. Therefore, this shift is the same in the reference fiber and the structure under test.

However, the fibers of different diameters exchange heat with the environment at different speeds. For this reason, the usage of the reference fiber of different radius restricts the spectral range of temperature drift that can be canceled out with this technique. Assuming that the rates of heat relaxation processes 
inside the fibers are much lower than the rates of the heat exchange between the fibers and the environment, one can express the temperature drifts of the fibers $T_{1,2}(t)$ for a given time dependence of the environment temperature $T_{\text {env }}(t)$ as

$$
T_{1,2}(t)=e^{-k_{1,2} t}\left[T_{1,2}^{(0)}+k_{1,2} \int_{0}^{t} e^{k_{1,2} \xi} T_{\text {env }}(\xi) \mathrm{d} \xi\right],
$$

where $T_{1,2}^{(0)}$ are the initial temperatures of the fibers and $k_{1,2}$ are the heat transfer coefficients at the fiber-air interface. If the fibers are identical $\left(k_{1}=k_{2}\right)$, their temperatures remain the same; if the fibers are different, their temperatures remain the same only if the characteristic time of ambient temperature drift, $\tau_{\text {env }}$, is much greater than the characteristic fiber heat relaxation times, $\tau_{\text {env }}>>\left(1 / k_{1,2}\right)$. The values of $k_{1,2}$ can be expressed as

$$
k=\frac{h S_{1,2}}{C_{1,2}}=\frac{4 h}{\rho c_{p} d_{1,2}},
$$

where $\rho \approx 2.2 \mathrm{~g} / \mathrm{cm}^{3}$ and $c_{p}=740 \mathrm{~J} /(\mathrm{kg} \cdot \mathrm{K})$ are the mass density and the specific heat of the material, respectively (values are given for fused silica), $C$ is the total heat capacity of the fiber, $S$ is its surface area, $h \approx 10 \mathrm{~W} /\left(\mathrm{m}^{2} \cdot \mathrm{K}\right)$ is the heat transfer coefficient between the fiber and the environment and $d$ is the diameter of the fiber. In the experiment, $d_{1}=38 \mu \mathrm{m}$ for SNAP fiber and $d_{2}=125 \mu \mathrm{m}$ for the reference fiber. This gives $\tau_{1} \approx 5 \mathrm{~s}$ and $\tau_{2} \approx 1.5 \mathrm{~s}$, which is much smaller than $\tau_{\text {env }} \gtrsim 5 \mathrm{~min}$ in our setup.

The proposed method will lead to an increase of precision only if the resonance drift during a single measurement is much smaller than the drift between consecutive scans. In order to check this, resonant wavelengths of two different WGMs of the reference fiber are measured in a separate experiment and the difference between them is calculated. In our experiments, the time taken by a single measurement of the spectrum is about $\approx 0.5 \mathrm{~s}$, while the period of time between two consecutive measurements is $\approx 10 \mathrm{~s}$. The observed difference $\lambda_{r 1}-\lambda_{r 2}$ does not exceed the value of the single step in wavelength of the spectrum analyzer of $1.6 \mathrm{pm}$ for all scans. The value of standard deviation of the difference $\left(\lambda_{r 1}-\lambda_{r 2}\right)$ is $\sigma=0.7 \mathrm{pm}$. This gives the reduction of the noise by an order of magnitude, as the deviations of absolute values of each of the resonant wavelengths are of the order of several picometers. The resulting precision of ERV characterization of SNAP is $\sigma r_{1} / \lambda_{r}=0.2 \AA$ over the whole characterization length.

The precision of measurements is also determined by the uniformity of the reference fiber along its axis. Relatively small and smooth ERV of the reference fiber allows one to characterize it by measuring spectra with lower spatial resolution as compared to the one required for the characterization of SNAP structures. Usually [8] the average slope of the ERV of a regular $125 \mu \mathrm{m}$ diameter single-mode fiber is $\sim 1 \mathrm{~nm}$ per $1 \mathrm{~mm}$ fiber length. In our experiments we look for the reference fiber segment with the smallest ERV, which is found to be less than $0.4 \mathrm{~nm}$ over 0.8 $\mathrm{mm}$ distance. The surface plot of the transmission amplitude of the reference fiber measured along the reference fiber with spatial resolution of $10 \mu \mathrm{m}$ is shown in Fig. 2. Compared to the characterization of the SNAP structure under test (spatial resolution of $2 \mu \mathrm{m}$ ), this characterization takes 5 times less time. This leads to the reduction of the thermal drift of the resonant frequencies originating from the slow change of the ambient temperature. To minimize the impact of the abrupt jumps associated with the technical noise of the spectrum analyzer and

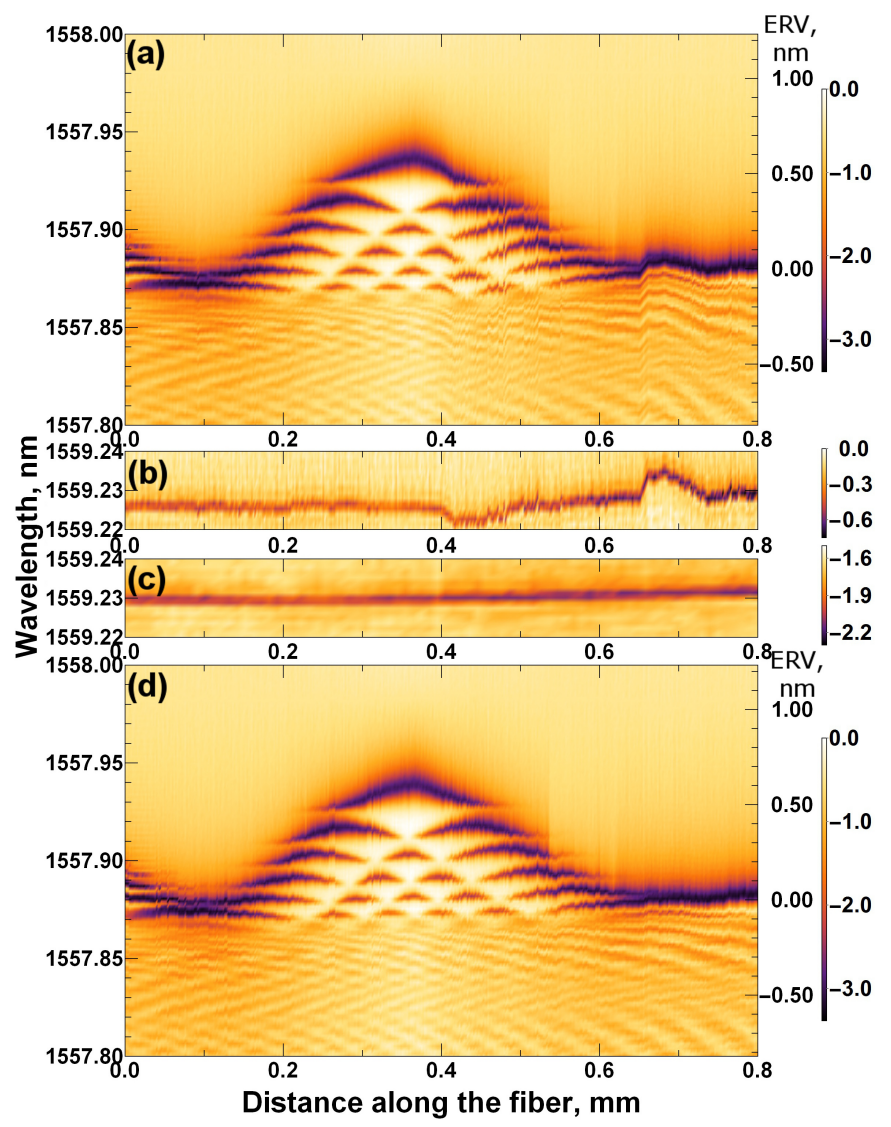

Fig. 3. Surface plots of the transmission amplitudes of: (a) a SNAP bottle resonator, measured; (b) a reference fiber, measured; (c) a reference fiber, calibration (Fig. 2); (d) a SNAP bottle resonator, corrected.

random dust-induced fluctuations of the coupling between the fiber and the microfiber, the scanning is performed several times and the results are averaged then. Such averaging is not suitable for the SNAP structure under test because it requires a significant increase of the number of spectral measurements, leading to degradation of the fiber surface due to many physical contacts with the microfiber.

The precision of characterization is also affected by the wavelength discretization of the spectrum analyzer if it is comparable to or exceeds the amplitude of the spectral noise. In order to reduce this effect, before averaging each of $i$ obtained dependencies of the reference resonant wavelength $\lambda_{r}^{(i)}(z)$ on the distance along the fiber $z$ is smoothed by moving average technique [17]. To exclude the influence of the temperature drift between the consecutive scans, the average value of $i$ several measurements of the resonant wavelength deviation $\Delta \lambda_{r}^{(i)}(z)=\lambda_{r}^{(i)}(z)-\lambda_{r}^{(i)}(0)$ is calculated as

$$
\Delta \lambda_{r}(z)=\overline{\lambda_{r}^{(i)}(z)-\left\langle\lambda_{r}^{(i)}\right\rangle}
$$

where the angle brackets represent the mean value over the whole measurement distance along the fiber equal to $0.8 \mathrm{~mm}$ (Fig. 2), while the overline means that the averaging is performed over $i$ different scans. The curve $\Delta \lambda_{r}(z)$ is a baseline for the calculation of the frequency shifts. This curve is plotted together with the error bars in Fig. 2. The averaging is performed over $i=3$ measurements. 
To illustrate the proposed method, a SNAP bottle resonator is fabricated and characterized. The resonator is introduced by a $1 \mathrm{~s}$ exposure of the fiber surface to a focused light beam with central wavelength of $10.6 \mu \mathrm{m}$ emitted from a $\mathrm{CO}_{2}$ laser (model Synrad 48-2). The surface plot of its transmission amplitude measured with the spatial resolution of $2 \mu \mathrm{m}$, is shown in Fig. 3(a). Fig. 3(b) shows the simultaneously measured spectra of the reference WGM. The difference between these spectra and calibration spectra of the reference fiber (shown in Fig. 3(c)) contains information about the measurement error of transmission amplitude of the structure under test. The corrected surface plot of the transmission amplitude of the structure under test is shown in Fig. 3(d). It is obtained by shifting the wavelengths of experimental data in Fig. 3(a) by the change of the resonant wavelength of the reference fiber shown in Fig. 3(b) with respect to the calibration Fig. 3(c). The remaining noise is caused by factors that cannot be taken into account by such referencing (see below).

Generally, the proposed method allows for reducing the influence of the factors that (i) contribute to the spectra of the reference fiber and of the fiber under test equivalently and (ii) affect them slowly, i.e. the spectral changes introduced during a single spectrum measurement must be small. In particular, these factors include: ambient temperature drift, technical frequency noise associated with the measuring device - spectrum analyzer and degradation of the microfiber surface. However, the proposed correction method does not take into account the factors that (i) contribute to the spectra of the reference fiber and the fiber under test differently or (ii) introduce significant changes to the spectrum during a single measurement. Among these factors are: the high-frequency noise of the measuring device and other fast random technical errors; contamination, degradation and charging of surfaces of the structure under test and the reference fiber.

The influence of the amplitude noise of the measuring device can be decreased by averaging over several separate spectral measurements taken for each point along $z$. Systematic errors (see, e.g., slow relaxation of the measurement reading after measuring a narrow peak in Fig. 3(a) at $0.5 \mathrm{~mm} \lesssim z \lesssim 0.65 \mathrm{~mm}$ ) can be reduced if necessary by choosing an appropriate device for spectral measurements. Alternatively, the time of a single spectrum measurement can be increased, but this will result in a trade-off between the decrease of this systematic error and increase of the lower limit of the spectral region in which the proposed method is effective for slow temperature drift.

After the elimination of aforementioned noise sources, random fluctuations of the coupling strength and losses become the main factors that determine the precision of SNAP characterization. These fluctuations are likely to be caused by contact electrification of the fiber and the microfiber when they are put into mechanical contact. The random changes of the surface electrical charge on the fiber and the microfiber affect the light propagation through these structures, bend the microfiber and attract dust particles from the environment to the fiber-microfiber interface electrostatically. While typical residual surface charge density of the $1.6 \mu \mathrm{m}$-diameter microfiber is about $4 \times 10^{-6} \mathrm{C} / \mathrm{m}^{2}$ [18], after direct contact this value may increase to the level of $10^{-4} \mathrm{C} / \mathrm{m}^{2}$ by the order of magnitude $[19,20]$. Improvement of the SNAP characterization scheme to reduce the impact of contact electrification lies beyond the scope of this letter. However, an obvious approach is to avoid the direct mechanical contact between the fiber and the microfiber at all. In this case the microfiber should be translated along the struc- ture under test axis at fixed distance from its surface. This can be achieved, for example, with locking the optical coupling by means of a piezoelectrically-driven feedback loop that corrects the distance between the fiber under test and the microfiber [21].

Apart from high-precision spectroscopy of SNAP structures, the method described above is applicable for control of the shape of tunable SNAP structures. Particularly, the position of the reference peak can be used as an input signal for feedback loops that lock the parameters of transient SNAP structures introduced mechanically [6] or by heating of an optical fiber [3].

In summary, a method of significant reduction of the technical noise associated with temperature drift of spectra and the frequency noise of the spectrum analyzer on the characterization of SNAP structures has been proposed, analyzed and demonstrated experimentally. The method is based on simultaneous coupling of a tapered microfiber to a SNAP structure under test and to a reference WGM of a regular optical fiber. The achieved precision of the measurement of the effective radius variation of the SNAP structure under test has been found to be of $0.2 \AA$.

Funding. Royal Society (WM130110), Horizon 2020 (H2020EU.1.3.3, 691011).

Acknowledgments. MS acknowledges the Royal Society Wolfson Research Merit Award.

\section{REFERENCES}

1. M. Sumetsky and Y. Dulashko, Optics express 20, 27896 (2012).

2. F. Shen, X. Shu, L. Zhang, and M. Sumetsky, Optics Letters 41, 2795 (2016).

3. A. Dmitriev, N. Toropov, and M. Sumetsky, "Transient reconfigurable subangstrom-precise photonic circuits at the optical fiber surface," in "2015 IEEE Photonics Conference (IPC)," (IEEE, 2015), pp. 1-2.

4. N. Toropov and M. Sumetsky, Optics letters 41, 2278 (2016).

5. M. Sumetsky, Physical review letters 111, 163901 (2013).

6. A. V. Dmitriev and M. Sumetsky, Optics letters 41, 2165 (2016).

7. T. Birks, J. Knight, and T. Dimmick, IEEE Photonics Technology Letters 12, $182(2000)$.

8. M. Sumetsky and Y. Dulashko, Optics letters 35, 4006 (2010).

9. M. Sumetsky, Scientific reports 5 (2015).

10. E. F. Burmeister, D. J. Blumenthal, and J. E. Bowers, Optical Switching and Networking 5, 10 (2008).

11. W. Bogaerts, M. Fiers, and P. Dumon, Selected Topics in Quantum Electronics, IEEE Journal of 20, 1 (2014).

12. W. Weng, J. D. Anstie, T. M. Stace, G. Campbell, F. N. Baynes, and A. N. Luiten, Physical review letters 112, 160801 (2014).

13. A. A. Savchenkov, A. B. Matsko, V. S. Ilchenko, N. Yu, and L. Maleki, JOSA B 24, 2988 (2007).

14. S. B. Papp, K. Beha, P. Del'Haye, F. Quinlan, H. Lee, K. J. Vahala, and S. A. Diddams, Optica 1, 10 (2014).

15. R. Matthey, F. Gruet, S. Schilt, and G. Mileti, Optics letters 40, 2576 (2015).

16. J.-J. Xiong, Y.-Z. Yan, C.-L. Zou, F.-W. Sun, S.-B. Yan, C.-Y. Xue, J. Liu, and W.-D. Zhang, "A stable frequency references platform based on packaged microsphere-taper coupling system," in "Nano/Micro Engineered and Molecular Systems (NEMS), 2012 7th IEEE International Conference on," (IEEE, 2012), pp. 335-338.

17. E. W. Weisstein, "Moving average," From MathWorld—A Wolfram Web Resource. http://mathworld.wolfram.com/MovingAverage.html.

18. K. Kamitani, T. Muranaka, H. Takashima, M. Fujiwara, U. Tanaka, S. Takeuchi, and S. Urabe, Optics Express 24, 4672 (2016).

19. W. Hu, L. Xie, and X. Zheng, Applied Physics Letters 101, 114107 (2012).

20. S. R. Waitukaitis, V. Lee, J. M. Pierson, S. L. Forman, and H. M. Jaeger, Physical Review Letters 112, 218001 (2014).

21. S. Spillane, T. Kippenberg, O. Painter, and K. Vahala, Physical Review Letters 91, 043902 (2003). 


\section{FULL REFERENCES}

1. M. Sumetsky and Y. Dulashko, "Snap: Fabrication of long coupled microresonator chains with sub-angstrom precision," Optics express 20, 27896-27901 (2012).

2. F. Shen, X. Shu, L. Zhang, and M. Sumetsky, "Fabrication of surface nanoscale axial photonics structures with a femtosecond laser," Optics Letters 41, 2795-2798 (2016).

3. A. Dmitriev, N. Toropov, and M. Sumetsky, "Transient reconfigurable subangstrom-precise photonic circuits at the optical fiber surface," in "2015 IEEE Photonics Conference (IPC)," (IEEE, 2015), pp. 1-2.

4. N. Toropov and M. Sumetsky, "Permanent matching of coupled optical bottle resonators with better than $0.16 \mathrm{ghz}$ precision," Optics letters 41, 2278-2281 (2016).

5. M. Sumetsky, "Delay of light in an optical bottle resonator with nanoscale radius variation: dispersionless, broadband, and low loss," Physical review letters 111, 163901 (2013).

6. A. V. Dmitriev and M. Sumetsky, "Tunable photonic elements at the surface of an optical fiber with piezoelectric core," Optics letters 41, 2165-2168 (2016).

7. T. Birks, J. Knight, and T. Dimmick, "High-resolution measurement of the fiber diameter variations using whispering gallery modes and no optical alignment," IEEE Photonics Technology Letters 12, 182-183 (2000).

8. M. Sumetsky and Y. Dulashko, "Radius variation of optical fibers with angstrom accuracy," Optics letters 35, 4006-4008 (2010).

9. M. Sumetsky, "Microscopic optical buffering in a harmonic potential," Scientific reports 5 (2015).

10. E. F. Burmeister, D. J. Blumenthal, and J. E. Bowers, "A comparison of optical buffering technologies," Optical Switching and Networking 5, 10-18 (2008).

11. W. Bogaerts, M. Fiers, and P. Dumon, "Design challenges in silicon photonics," Selected Topics in Quantum Electronics, IEEE Journal of 20, 1-8 (2014).

12. W. Weng, J. D. Anstie, T. M. Stace, G. Campbell, F. N. Baynes, and A. N. Luiten, "Nano-kelvin thermometry and temperature control: beyond the thermal noise limit," Physical review letters 112, 160801 (2014).

13. A. A. Savchenkov, A. B. Matsko, V. S. Ilchenko, N. Yu, and L. Maleki, "Whispering-gallery-mode resonators as frequency references. ii. stabilization," JOSA B 24, 2988-2997 (2007).

14. S. B. Papp, K. Beha, P. Del'Haye, F. Quinlan, H. Lee, K. J. Vahala, and S. A. Diddams, "Microresonator frequency comb optical clock," Optica 1, 10-14 (2014).

15. R. Matthey, F. Gruet, S. Schilt, and G. Mileti, "Compact rubidiumstabilized multi-frequency reference source in the $1.55-\mu \mathrm{m}$ region," Optics letters 40, 2576-2579 (2015).

16. J.-J. Xiong, Y.-Z. Yan, C.-L. Zou, F.-W. Sun, S.-B. Yan, C.-Y. Xue, J. Liu, and W.-D. Zhang, "A stable frequency references platform based on packaged microsphere-taper coupling system," in "Nano/Micro Engineered and Molecular Systems (NEMS), 2012 7th IEEE International Conference on," (IEEE, 2012), pp. 335-338.

17. E. W. Weisstein, "Moving average," From MathWorld—A Wolfram Web Resource. http://mathworld.wolfram.com/MovingAverage.html.

18. K. Kamitani, T. Muranaka, H. Takashima, M. Fujiwara, U. Tanaka, S. Takeuchi, and S. Urabe, "Measuring the charge density of a tapered optical fiber using trapped microparticles," Optics Express 24, 46724679 (2016).

19. W. Hu, L. Xie, and X. Zheng, "Contact charging of silica glass particles in a single collision," Applied Physics Letters 101, 114107 (2012).

20. S. R. Waitukaitis, V. Lee, J. M. Pierson, S. L. Forman, and H. M. Jaeger, "Size-dependent same-material tribocharging in insulating grains," Physical Review Letters 112, 218001 (2014).

21. S. Spillane, T. Kippenberg, O. Painter, and K. Vahala, "Ideality in a fibertaper-coupled microresonator system for application to cavity quantum electrodynamics," Physical Review Letters 91, 043902 (2003). 